\title{
Evaluation of APeLS - An Adaptive eLearning Service based on the Multi-model, Metadata-driven Approach
}

\author{
Owen Conlan and Vincent Wade \\ Knowledge and Data Engineering Group \\ Trinity College, Dublin Ireland \\ \{Owen. Conlan, Vincent.Wade\}@cs.tcd.ie \\ http://kdeg.cs.tcd.ie
}

\begin{abstract}
The evaluation of learner and tutor feedback is essential in the production of high quality personalized eLearning services. There are few evaluations available in the Adaptive Hypermedia domain relative to the amount of research interest this domain is attracting. Many of the papers in this domain focus on the technological design of systems without justifying the designs through the lessons learned from evaluations. This paper evaluates the usability and effectiveness of using the multi-model, metadata-driven approach for producing rich adaptive eLearning solutions that remain content and domain independent. Through this independence, the eLearning services developed can utilize many pedagogical approaches and a variety of models to produce a wide range of highly flexible solutions. This paper identifies benefits to learners brought through adopting the multi-model approach gathered over four years of student evaluation. It briefly describes the evaluation of the Adaptive Personalized eLearning Service (APeLS), a personalized eLearning service based on a generic adaptive engine.
\end{abstract}

\section{Introduction}

Adaptive content systems are generally either systems that provide high levels of adaptivity on a specific content domain, such as ELM-ART II [4] or are generic adaptive systems that provide fundamental levels of adaptivity on non-specific domains AHA! [2]. A general problem with the application of many Adaptive Hypermedia systems to the area of eLearning is that the role of pedagogy is often undermined or ignored. Twenty years of using ICT for learning has shown repeatedly that favoring technological solutions over pedagogical soundness results in beautifully crafted systems that are unusable.

Traditionally adaptive eLearning systems are not independent from the content they adapt. This is due to the fact that the deeper the pedagogical strategies and axes of adaptivity are embedded the more tied to the specific con-tent and learning objects they become. By intertwining the mechanisms that provide the adaptive effects with the content that is being operated on many adaptive applications achieve high levels of adaptivity through manipulating the content. The sacrifice in this approach is that the content, which is often expensive and time-intensive to develop, is not easily 
reused. Similarly, it becomes difficult, if not impossible, to apply different pedagogical approaches in the personalization of the content. Conversely, many of the nondomain specific adaptive solutions sometimes facilitate the reuse of content. However, this reuse often comes at the cost of limiting the adaptive effects to light-weight approaches such as conditional fragments and link hiding.

Over the past four years the Adaptive Personalized eLearning Service (APeLS) [1] has been used as a personalized teaching tool as part of undergraduate degree level courses in Trinity College, Dublin. The development and improvement of this application has been based on the evolving Adaptive Engine (AE) at its core. The multi-model, metadata-driven approach, which aims to alleviate many the issues described above, is the methodology used in the design of the AE and APeLS. The use of this adaptive system has been evaluated over this period and some of the findings from the student evaluation are presented in this paper.

\section{Trial and Evaluation}

In Trinity College, Dublin (TCD) SQL is taught as part of seven degree programs across ten different courses. Each course differs based on the degree focus and ethos, objectives, student experience and complexity. Each of these courses have different subject emphasis and different lecturers responsible for teaching on the courses. Junior and Senior Sophister (3rd and 4th year) students in four degree courses degree courses at TCD utilize a personalized SQL (Structured Query Language) course as part of their studies on data storage and database design. A goal of the personalized SQL course is to facilitate the teaching of Structured Query Language as part of these diverse courses, supporting different objects, pedagogical styles, prior knowledge and academic scope. In 1998 and 1999 a non-adaptive SQL online resource, [3] also developed by TCD, was blended into the teaching of the database courses for the Computer Science and Computer Science Language and Linguistic students. This nonadaptive resource offered students a structured online course in SQL with supplementary features such as a live database that they could query via a web browser interface, a detailed case study that formed the basis of many of the examples found in the course and a project reference section.

Using the principles described in the multi-model, metadata driven approach [1] this non-adaptive SQL course was chosen as the basis of a personalized eLearning course in SQL. The multi-model approach describes the necessity for at least three separate and discrete models - learner, content and narrative. It is important to note that the curriculum of the course did not change with the introduction of the personalized eLearning service and that the content used in the personalized service is based directly on that of the original non-adaptive course. The personalized course on SQL is an adaptable, competencies-based course comprised of a didactic pedagogical narrative, over 330 fine-grained pagelets and a tutor-developed competency ontology of 92 terms that forms the domain model. 
Table 1. Summary of Student Evaluation Questionnaires, 2000 - 2003

\begin{tabular}{|c|c|c|c|c|}
\hline Question $\quad$ Response & None & Little & Some & Much \\
\hline $\begin{array}{l}\text { 1) How much experience in SQL did } \\
\text { you have before commencing the } \\
\text { online SQL course? }\end{array}$ & $29 \%$ & $38 \%$ & $27 \%$ & $6 \%$ \\
\hline $\begin{array}{l}\text { 2) How much experience did you } \\
\text { have using online learning resources? }\end{array}$ & $49 \%$ & $19 \%$ & $17 \%$ & $15 \%$ \\
\hline & Never & Rarely & Usually & Always \\
\hline $\begin{array}{l}\text { 3) Did the course(s) generated reflect } \\
\text { the answers you gave in the knowl- } \\
\text { edge pre-test instrument? }\end{array}$ & $2 \%$ & $7 \%$ & $58 \%$ & $33 \%$ \\
\hline $\begin{array}{l}\text { 4) Would you have liked more con- } \\
\text { trols over the content included in the } \\
\text { personalized courses? }\end{array}$ & $23 \%$ & $38 \%$ & $28 \%$ & $11 \%$ \\
\hline $\begin{array}{l}\text { 5) Did the course(s) generated by the } \\
\text { system reflect the course(s) you } \\
\text { wanted? }\end{array}$ & $3 \%$ & $18 \%$ & $63 \%$ & $16 \%$ \\
\hline $\begin{array}{l}\text { 6) Were the courses generated easy to } \\
\text { navigate? }\end{array}$ & $0 \%$ & $10 \%$ & $59 \%$ & $31 \%$ \\
\hline $\begin{array}{l}\text { 7) Was the quantity of content on } \\
\text { each page satisfactory? }\end{array}$ & $6 \%$ & $25 \%$ & $61 \%$ & $8 \%$ \\
\hline $\begin{array}{l}\text { 8) Would you have liked a greater } \\
\text { level of control as to how the content } \\
\text { was structured? }\end{array}$ & $40 \%$ & $47 \%$ & $11 \%$ & $2 \%$ \\
\hline
\end{tabular}

The evaluations have been carried out over the past four years (2000 - 2003, inclusive) and include two separate evaluations - student and tutor. The student evaluation, which is the focus of this paper, was carried out on over 500 students and attempts to determine the usability of the personalized SQL course, in particular focusing on learner satisfaction and the effectiveness of the service. Each of the students was presented with the same paper-based questionnaire giving them an opportunity to evaluate the personalized SQL course they had just completed. The questionnaire comprised thirty one mostly multiple choice questions (some of which are represented in Table 1, above) and an open comments section. Each question also had space for the student to elaborate on their answers.

Also as part of the evaluation of the personalized SQL course the effectiveness of the course was assessed by examining the students examination performance over the period of the evaluation (2000 - 2003) and for the previous two years when an online non-adaptive (based on the same content) version of the SQL was used (Fig. 1, above). The effectiveness of the personalized SQL course is examined by comparing it across to two different axes - how students performed using the non-adaptive online courses prior to the introduction of the personalized course and how students performed in examination questions related and not related to the material presented in the online SQL course. The former metric gives some historical perspective on the impact of the adaptive versus a non-adaptive course across different peer groups, 
while the latter metric shows how in the same peer groups the adaptive course affected their performance.

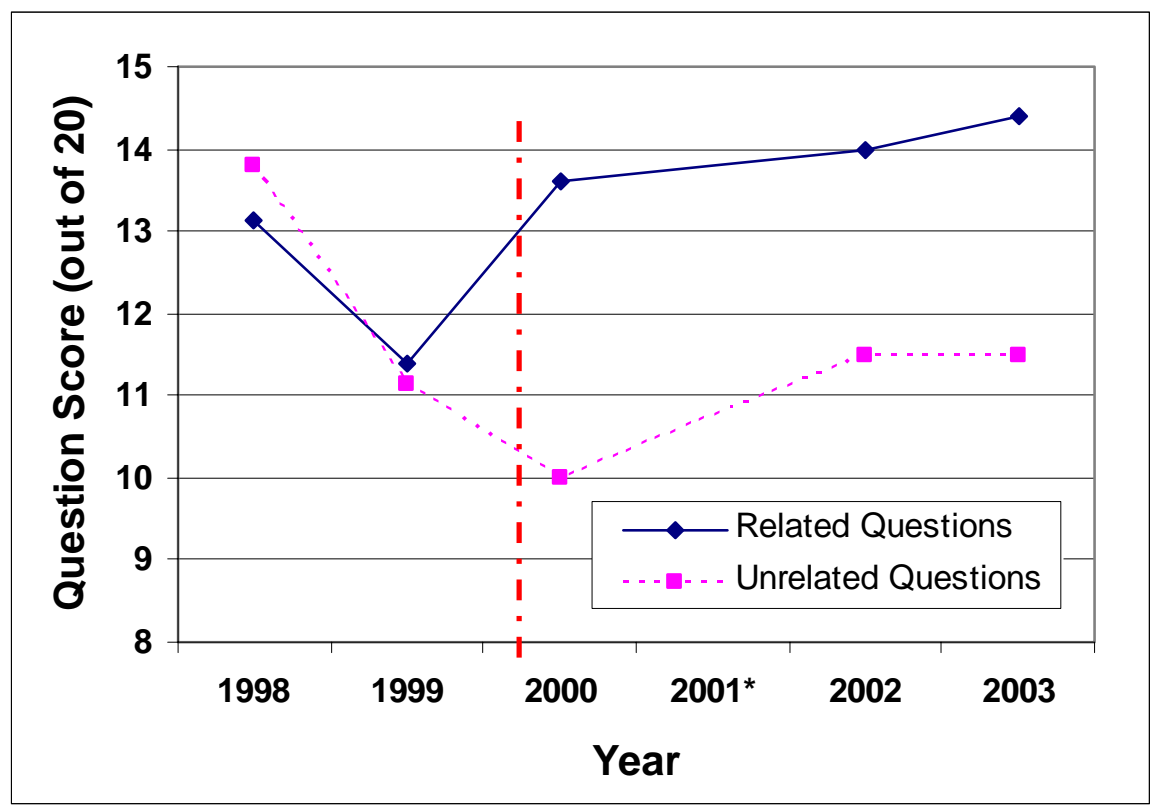

Fig. 1. Average Question Scores on Database Examinations, 1998 - 2003

Figure 1, above, summarizes the performance of six classes of final year students in two degree courses. Both of these courses receive a common lecture course, similar examinations and both use online tools to learn SQL. In 1998 and 1999 the online tools were non-adaptive. The personalized SQL course was first introduced in 2000 (signified by the vertical dotted line). The Question Score axis shows the average question score out of a possible twenty marks. The two lines shown on the graph represent the average question scores in questions related and unrelated to the material taught in the online SQL courses. The questions were not the same from year to year, but were similar and of comparable difficulty. ${ }^{*} 2001$ results are interpolated as statistics were.

\section{$3 \quad$ Initial Findings and Conclusion}

Initial findings from the student evaluation show that despite the majority of students having little or no experience of online learning or the subject matter (Table 1, Questions 1 \& 2) the introduction of the personalized SQL course in 2000 had a beneficial effect on students' performance in the final examinations (Figure 1). As may be seen from the graph the students in 1998 scored well in both questions related and unrelated to SQL, yet in the following year their scores declined, possibly indicating a weaker class of students. From 2000, with the introduction of the personalized SQL 
course, the scores in questions related to SQL have risen steadily and the scores in these questions display an increase of 2-3 marks over the questions unrelated to SQL. In a question worth 20 marks this represents approximately a $10-15 \%$ increase in performance by the students. This difference may be attributed to the benefits of the personalized SQL course, as the SQL content presented shared a common basis with that of the non-adaptive course.

Most students felt that the courses generated based on the prior knowledge pretest instrument produced courses they expected and desired (Questions $3 \& 5$ ), however approximately $40 \%$ of students stated that they desired more control over the course content (Question 4). This was reflected in the comments of a number of students where they stated that they 'played' the pre-test instrument to create short personalized courses that could be tackled in short periods of time. They would repersonalize the course for each session with this aim. This finding highlights the innovation some learners can display in utilizing personalized eLearning.

This paper has briefly described the evaluation process of a personalized SQL course based on the Adaptive Personalized Learning Service (APeLS) and presented some initial findings from this evaluation.

\section{References}

1. Conlan, O., Wade, V., Bruen, C., Gargan, M. (2002) Multi-Model, Metadata Driven Approach to Adaptive Hypermedia Ser-vices for Personalized eLearning. In the Proceedings of Second International Conference on Adaptive Hypermedia and Adap-tive Web-Based Systems, AH 2002, 100-111.

2. De Bra, P., Aerts, A., Berden, B., De Lange, B., Rousseau, B., Santic, T., Smits, D., Stash, N., (2003) AHA! The Adaptive Hypermedia Architecture. In the Proceedings of the ACM Hypertext Conference, Nottingham, UK, August 2003.

3. Wade, V., Power, C. (1998) Evaluating the design and delivery of WWW based educational environments and courseware. In the Proceedings of the 6th annual conference on the teaching of computing and the 3rd annual conference on Integrating tech-nology into computer science education, 243 - 248 .

4. Weber, G. and Brusilovsky, P. (2001) ELM-ART: An adaptive versatile system for Webbased instruction. International Jour-nal of Artificial Intelligence in Education 12 (4), Special Issue on Adaptive and Intelligent Web-based Educational Systems, 351-384. 